\title{
Sampling precautions for the measurement of nitrated polycyclic aromatic hydrocarbons in ambient air
}

\author{
A. Albinet ${ }^{a^{*}}$, E. Leoz-Garziandia ${ }^{a^{*}}$, H. Budzinski ${ }^{b}$, E. Villenave $^{c}$ \\ ${ }^{a}$ Institut National de l'Environnement industriel et des RISques (INERIS), Parc technologique Alata BP2, 60550 \\ Verneuil en Halatte, France \\ ${ }^{b}$ Laboratoire de Physico-\& Toxico Chimie des systèmes naturels (LPTC), UMR 5472 CNRS, Université Bordeaux 1, \\ 33405 Talence, France \\ ${ }^{c}$ Laboratoire de Physico-Chimie Moléculaire (LPCM), UMR 5803 CNRS, Université Bordeaux 1, 33405 Talence, \\ France \\ ${ }^{*}$ Corresponding authors. Fax : +33 344556302 \\ E-mail addresses: alexandre.albinet@gmail.com (A. Albinet), eva.leoz@ineris.fr (E.Leoz-Garziandia).
}




\section{Abstract}

Because of the toxicity of polycyclic aromatic hydrocarbons (PAHs) and of their oxidation products, such as nitrated and oxygenated PAHs (NPAHs and OPAHs), the determination of their concentrations is of great interest in terms of atmospheric pollution control. Then, normalisation of sampling procedures appears essential. In this context, this paper presents a comparison of particulate PAH, OPAH and NPAH concentrations determined with two different samplers (cascade impactor and conventional high volume sampler) installed in parallel during several field sampling campaigns carried out under different environmental conditions. For winter and summer periods, the PAH and OPAH concentrations determined with both sampling systems were considered as equivalent. In the summer period, NPAH concentrations quantified with both sampling devices were similar whereas in the winter period, the conventional high volume sampler underestimated their concentrations by a factor of 3 to 4 . This underestimation was observed in the same proportion for all the 17 quantified NPAHs. Analytical error, NPAH formation during the sampling and NPAH degradation by reaction with gaseous oxidants associated to sampling methodology were unable to explain such differences between both samplers used in parallel. A probable hypothesis is that, the heating of the $\mathrm{PM}_{10}$ head of the high volume sampler in the winter period generates an increase of the internal sampler temperature that could intensify the chemical degradation of the NPAHs inducing the underestimation of their concentrations in the atmosphere. Further investigations will be necessary to confirm the importance of the temperature on the chemical degradation of these compounds and to understand the different behaviour of PAHs and OPAHs. Consequently, we suggest using oxidant scrubber to prevent chemical degradation of PAHs and derivatives during their sampling. Moreover, we advise against the heating of the sampling head which could induce an increase of these reactions of degradation especially for NPAHs. 
Keywords: Polycyclic aromatic hydrocarbons; Nitrated polycyclic aromatic hydrocarbons; Oxygenated polycyclic aromatic hydrocarbons; Sampling artefacts

\section{Introduction}

Atmospheric aerosols are complex matrices which include different chemical species that could cause harmful effects on human health (Finlayson-Pitts and Pitts Jr, 2000). Polycyclic aromatic hydrocarbons (PAHs) are typical toxic compounds present in the particulate matter. Several PAH derivatives, such as nitrated PAHs (NPAHs) and oxygenated PAHs (OPAHs) having strong indirect- and direct-acting mutagenic properties, are also found in airborne particulate matter (Allen et al., 1997; Bamford and Baker, 2003). PAHs are emitted directly from combustion whereas NPAHs and OPAHs are both primarily emitted and formed in the atmosphere, by gas and heterogeneous phase reactions of PAHs induced by atmospheric oxidants $\left(\mathrm{OH}, \mathrm{NO}_{3}, \mathrm{O}_{3}\right)$. PAH derivatives have a particular interest because they seem more toxic than their related parent PAHs. For instance, NPAHs could contribute for $10 \%$ to the total mutagenicity of inhalable suspended particles in polluted areas (Arey et al., 1988b; Atkinson and Arey, 1994). A correct quantification of these compounds in ambient air is very important.

Some surprising results obtained in this study suggest that some precautions have to be taken for the measurement of the PAHs and their derivatives in the ambient air. 


\section{Experimental section}

\subsection{Sampling sites and operating conditions}

Experiments were carried out on four sampling sites located in the French Mediterranean and Alpine regions. Table 1 summarises dates, locations, main characteristics and atmospheric conditions during the campaigns.

The two sites located in the Alpine region (Chamonix and Maurienne valleys) were involved in the sampling campaigns of the POVA research program (POllution des Vallées Alpines) which focused on atmospheric chemistry in the vicinity of the most important automotive traffic between France and Italy. Four campaigns of one week each were carried out (two per site), in winter 20022003 and in summer 2003 (Table 1). Traffic related site named “Les Bossons” was instrumented in the Chamonix valley and a rural one in the small hamlet of Tigny in the Maurienne valley.

The two sites of the Mediterranean region were located in the Marseilles area (city of 800000 inhabitants) characterised by an important solar irradiation, heavy traffic and industrial activities. Two different types of sites (urban "Cinq avenues” and sub-urban "La Penne sur Huveaune “) were instrumented for the sampling of PAHs and their derivatives.

\subsection{Sampling procedure}

On each site, two kinds of samplers were used in parallel. Both ambient air particulate (quartz filter) and gas (PUF: polyurethane foams) phases were collected using a modified high volume sampler equipped with a $\mathrm{PM}_{10}$ head (Model Digitel DA-80, Hegnau, Switzerland, $30 \mathrm{~m}^{3} \mathrm{~h}^{-1}$ ). As recommended by the constructor, during the winter period the $\mathrm{PM}_{10}$ head was heated to prevent turbine overload due to moist filters because of the excessive ambient humidity. The high volume cascade impactor (Graseby Andersen, $35 \mathrm{~m}^{3} \mathrm{~h}^{-1}$ ) collecting airborne particulate was the second 
sampling device used. The particles collected using this sampler were separated into seven size ranges and collected on six separated fritted quartz fiber filters and on a back up quartz fiber filter. Samplings were performed every $12 \mathrm{~h}$ with the DA-80 sampler and every $24 \mathrm{~h}$ with the cascade impactor (starting at 08:00 local time).

\subsection{Analytical methods}

Detailed analytical procedure for PAHs, NPAHs and OPAHs has been presented previously Albinet et al., 2006. Filters and PUF were extracted with dichloromethane using pressurised liquid extraction. Extracts were divided into two fractions by weighting. Both fractions were evaporated under a nitrogen stream and adjusted to $1 \mathrm{ml}$ with acetonitrile (PAH analysis) or dichloromethane (NPAH and OPAH analysis). 16 PAHs were quantified by HPLC with fluorescence/UV detection (acenaphthene, fluorene, phenanthrene, anthracene, fluoranthene, pyrene, benz[a]anthracene, chrysene, benzo[e]pyrene, benzo[b]fluoranthene, benzo[k]fluoranthene, benzo[a]pyrene, dibenz[a,h]anthracene, benzo[g,h,i]perylene, indeno[1,2,3,c,d]pyrene and coronene). NPAH and OPAH concentrations were determined simultaneously using gas chromatography-mass spectrometry with negative ion chemical ionisation (GC/NICI-MS) after purification on solid phase extraction (SPE) cartridges. A total of 17 NPAHs and 8 OPAHs were quantified (1nitronaphthalene, 2-nitronaphthalene, 2-nitrofluorene, 9-nitroanthracene, 9-nitrophenanthrene, 3nitrophenanthrene, 3+2-nitrofluoranthene, 4-nitropyrene, 1-nitropyrene, 2-nitropyrene, 7nitrobenz[a]anthracene, 6-nitrochrysene, 1,3-dinitropyrene, 1,6-dinitropyrene, 1,8-dinitropyrene and 6-nitrobenzo[a]pyrene; 1-naphthaldehyde, 9-fluorenone, 9-phenanthrenecarboxaldehyde, 9,10anthraquinone, benzo[a]fluorenone, benzo[b]fluorenone, benzanthrone and benz[a]anthracene-7,12dione). 


\subsection{Quality assurance}

PAH analytical procedure was validated during a national inter-comparison campaign (LeozGarziandia, 2004). Three kinds of samples were analysed in order to identify the critical steps of the analytical protocols (low and high concentrations of prepared standard solutions, extracts of natural ambient air samples and diesel standard reference material 2975). For each sample four replicate analyses were carried out by each laboratory and mean values were calculated.

Application of the OPAH and NPAH analytical procedure has been performed on standard reference material (SRM 1649a: urban dust), giving results in good agreement with the few data available in the literature (Albinet et al., 2006).

\section{Results}

\subsection{Concentrations and sources of PAHs, OPAHs and NPAHs}

Average levels of concentrations observed during each sampling campaign for the sum of PAHs, OPAHs and NPAHs present mainly in particulate phase ( $\Sigma$ PAHp, $\Sigma$ OPAHp and $\Sigma$ NPAHp) and for the gaseous pollutants $\mathrm{NO}, \mathrm{NO}_{2}$ and $\mathrm{O}_{3}$ are presented on Table 1.

\subsection{Comparison of both sampling devices}

A comparison between particulate-bound individual PAH, OPAH and NPAH concentrations obtained with both samplers was made. Fig. 1 shows the results obtained by summing the concentrations of all the impactor stages and averaging the concentrations of both 12-hour total filtration samples (DA-80). Only compounds present mainly in the particulate phase were taking 
into account. All sampling sites were mixed and results were presented according to the sampling season (winter and summer).

In the summer period, for all compound families (PAHs, OPAHs and NPAHs) the correlation coefficients were quite good, indicating a good proportionality between both sampling methods. The intercepts were really low, indicating no systematic bias. The slopes of the regression were close to 1, indicating minimal (or at least similar) "blowoff" or adsorption artefacts. Thus, the concentrations determined with both sampling systems were considered as equivalent with regard to the analysis for particulate PAHs, OPAHs and NPAHs.

In the winter period, the same conclusion could be drawn for PAHs and OPAHs. The differences between the PAH and OPAH concentrations determined with both samplers were not significant. Concerning NPAHs, results were wholly different. Even if the correlation coefficient was good and the intercept quite low, the slope of the regression was significantly different from 1 . Individual NPAHs concentrations determined by the cascade impactor were about 3 to 4 times higher than those determined by the DA-80.

To investigate the origin of such differences, numerous hypotheses were tested: analytical drift, production or destruction of particle-bound NPAHs during the sampling. Each of these possibilities is discussed below.

\section{Discussion}

\subsection{Analytical drift}

The analytical procedure used in this study was validated on standard reference material (urban dust, SRM 1649a) and results previously published (Albinet et al., 2006) have shown a very repeatable method with a mean coefficient of variation (standard deviation/mean) about 0.2 . In this procedure, OPAHs and NPAHs were analysed simultaneously and so, if analytical drift occurred, it 
should be the same for both classes of compounds. Moreover, the greater discrepancies were obtained with the most concentrated samples (winter period) for which the analytical errors were minimal. The uncertainties of the analytical procedure cannot explain the important differences observed between both kinds of samplers used in winter.

\subsection{Formation of NPAHs during sampling}

The formation of NPAHs during sampling by heterogeneous reaction with nitrogen oxides was investigated in previous studies. During a winter high-NOx episode, the maximum percentage of artefact formation of the observed NPAHs was less than 2-3\% (Arey et al., 1988a). Results from a study conducted in a tunnel showed that the formation artefact represented less than $0.1 \%$ of the studied NPAHs (Dimashki et al., 2000). Authors concluded that filter artefacts were not significant and were not problematic for NPAH ambient air samplings. In our case, differences of concentrations of the parent PAHs determined by both sampling devices were not significant and differences of concentrations between the two samplers used were the same for any primary (e.g. 1-

nitropyrene) or secondary (e.g. 2-nitropyrene, 2+3-nitrofluoranthene) NPAHs. Moreover, if formation during sampling were to take place, that would be easier in the summer period when atmospheric concentrations of the reaction initiators, $\mathrm{OH}$ and $\mathrm{NO}_{3}$, were maximal. The formation of NPAHs during the sampling could not explain the disparities noted in the winter period between DA-80 and impactor samplers.

\subsection{Destruction of NPAHs during sampling}

The major way of degradation of NPAHs is photolysis. In both cases, this parameter could not be taken into account because the collection filter, installed in the middle of the sampler, was protected from sunlight. 
A second way of degradation of particulate NPAHs by reaction with gaseous oxidants such as, $\mathrm{OH}, \mathrm{O}_{3}$ and $\mathrm{NO}_{\mathrm{x}}$ was reported as very negligible (Atkinson et al., 1989; Feilberg et al., 1999). Due to the difference of the sampling methodology used in this study, this way of degradation could play a role in the differences observed. Because of the conditions of contact between ambient air and particles in the impactor, there is hypothetically a greater probability of contact with the compounds (NPAHs) on the filtration unit (backup filter) of the impactor-a condition that would also exist for the DA-80 filters. However, in the cascade impactor, airflow during sampling is over the surface of the fritted filters. It is conceivable that only the upper outside surface of the top layer of the impacted particles on the fritted filters is exposed to potential oxidizing agents, whereas all sampled air must pass through the backup filter. For the summer samples from the Alpine region, the percentage of the NPAHs collected on the fritted filters reached 85\% (Fig. 2). In this case, if chemical reaction with atmospheric oxidants was a major way of degradation of the NPAHs collected on the filtration unit, the NPAH concentrations determined with the impactor would be much more important than those given by the classical filter method (DA-80). As show in Table 1, it is not the case. On the opposite, when the differences of concentrations determined by both samplers were the greatest in the winter period, the NPAHs were mainly associated to the finest particles collected on the back up filter. Finally, during the summer sampling campaign in the region of Marseilles, no or slight differences of concentrations were observed between the samplers whereas the NPAH filter distribution was similar to that of the winter samples from the Alpine region. Degradation of particulate NPAHs on the filter by reaction with gaseous oxidants associated to sampling methodology does not seem to be the key factor explaining the differences of concentrations observed between both samplers used in parallel.

The difference of temperature between external and internal ambient air could play a role in the differences of NPAH concentrations observed between both samplers used. The particulate collection filters of the DA-80 are located in the body of the sampler. The heating of the $\mathrm{PM}_{10}$ head 
used in winter and to a lesser extent, the heat emitted by the sampling turbine did increase the internal temperature of the DA-80 sampler (up to $25^{\circ} \mathrm{C}$ more). Note that the increase of the internal temperature of the DA-80 did not cause the volatilisation of the particulate compounds collected on the filter. In fact, the percentage of PAHs, OPAHs and NPAHs with molecular weight $>206 \mathrm{~g} \mathrm{~mol}^{-1}$ associated with the particulate phase was in the range of $90-100 \%$ during the winter period. In summer, the heating of the $\mathrm{PM}_{10}$ head was not used and the difference between internal and external temperatures was lower (maximum difference observed about $10^{\circ} \mathrm{C}$ ). In the case of the impactor, collection filters are located on the upper part of the sampler. The pump is external and the sampler is well ventilated. The external and internal temperatures at the level of the collection filters were approximately similar in wintertime. Fig. 3 shows clearly the importance of the difference of internal and external temperature of the DA-80 sampler on the determination of NPAH concentrations. For any difference between internal DA-80 and ambient air temperatures, PAH and OPAH concentration ratios were in the range of 0.5-2. For NPAHs, this ratio increased considerably until a value of 9 when differences of temperature were the greatest. At this time, we are unable to explain with certitude differences observed on the NPAH behaviour in comparison with the PAHs and OPAHs. A probable hypothesis to explain such differences is that the abrupt change of temperature between external ambient air (negative temperature in winter) and inside the DA-80 sampler could intensify the chemical degradation of the NPAHs. In the same time, the lower temperature in the impactor sampler stabilises these compounds collected on the filters. Temperature does not seem to play a role in the degradation of the other classes of compounds namely PAHs and OPAHs. Nevertheless, as far as we know no data, showing the relation (or not) between chemical degradation of PAHs and their derivatives and the increase of the temperature permitting to confirm our hypothesis, exist. 


\section{Conclusion}

This study presents a comparison of particulate PAH, OPAH and NPAH concentrations determined with two kinds of samplers (cascade impactor and conventional high volume sampler) installed in parallel during five sampling campaigns. For both winter and summer periods, the PAH and OPAH concentrations determined with both sampling systems were considered as equivalent with regard to the analysis of these compounds. In the summer period, NPAH concentrations quantified with both sampling devices were similar whereas in the winter period, the DA-80 sampler underestimated their concentrations by a factor of 3 to 4 . To investigate the origin of such differences numerous hypotheses were tested: analytical drift, production or destruction of particlebound NPAHs during the sampling. Finally, a probable hypothesis is that, the heating of the DA-80 $\mathrm{PM}_{10}$ head in the winter period generates an increase of the internal temperature of the sampler that could intensify the chemical degradation of the NPAHs inducing the underestimation of their concentrations in the atmosphere. Further investigations will be necessary to confirm the importance of the temperature on the chemical degradation of these compounds and the different behaviour of PAHs and OPAHs. As a conclusion and as suggested in a previous study (Goriaux et al., 2006), the use of oxidant scrubber would allow to prevent chemical degradation of PAHs and derivatives during their sampling. Moreover, we advise against the heating of the sampling head which could induce an increase of these reactions of degradation especially for NPAHs.

\section{Acknowledgements}

The authors wish to thank the French Ministry of Ecology and Sustainable Development (MEDD) and the ADEME agency for financial support. 


\section{References}

Albinet, A.,Leoz-Garziandia, E.,Budzinski, H. and ViIlenave, E., 2006. Simultaneous analysis of oxygenated and nitrated polycyclic aromatic hydrocarbons on standard reference material 1649a (urban dust) and on natural ambient air samples by gas chromatography-mass spectrometry with negative ion chemical ionisation. Journal of Chromatography A 1121, 106-113.

Allen, J. O.,Dookeran, N. M.,Taghizadeh, K.,Lafleur, A. L.,Smith, K. A. and Sarofim, A. F., 1997. Measurement of oxygenated polycyclic aromatic hydrocarbons associated with a size-segregated urban aerosol. Environmental Science \& Technology 31, 2064-2070.

Arey, J.,Zielinska, B.,Atkinson, R. and Winer, A. M., 1988a. Formation of nitroarenes during highvolume sampling. Environmental Science \& Technology 22, 457-462.

Arey, J.,Zielinska, B.,Harger, W. P.,Atkinson, R. and Winer, A. M., 1988b. The contribution of nitrofluoranthenes and nitropyrenes to the mutagenic activity of ambient particulate organic matter collected in Southern California. Mutation Research 207, 45-51.

Atkinson, R.,Aschmann, S. M.,Arey, J.,Zielinska, B. and Schuetzle, D., 1989. Gas-phase atmospheric chemistry of 1- and 2-nitronaphtalene and 1,4-naphtoquinone. Atmospheric Environment 23, 2679-2690.

Atkinson, R. and Arey, J., 1994. Atmospheric chemistry of gas-phase polycyclic aromatic hydrocarbons: formation of atmospheric mutagens. Environmental Health Perspectives 102, 117126.

Bamford, H. A. and Baker, J. E., 2003. Nitro-polycyclic aromatic hydrocarbon concentrations and sources in urban and suburban atmospheres of the Mid-Atlantic region. Atmospheric Environment 37, 2077-2091. 
Dimashki, M.,Harrad, S. and Harrison, R. M., 2000. Measurements of nitro-PAH in the atmospheres of two cities. Atmospheric Environment 34, 2459-2469.

Feilberg, A.,Kamens, R. M.,Strommen, M. R. and Nielsen, T., 1999. Modeling the formation, decay, and partitioning of semivolatile nitro-polycyclic aromatic hydrocarbons (nitronaphthalenes) in the atmosphere. Atmospheric Environment 33, 1231-1243.

Finlayson-Pitts, B. J. and Pitts Jr, J. N., 2000. Chemistry of the upper and lower atmosphere. WileyInterscience: 969 p.

Goriaux, M.,Jourdain, B.,Temime, B.,Besombes, J.-L.,Marchand, N.,Albinet, A.,Leoz-Garziandia, E. and Wortham, H., 2006. Field comparison of particulate PAH measurements using a low flow denuder device and conventional sampling systems. Environmental Science \& Technology 40, 6398-6404.

Leoz-Garziandia, E., 2004. Essais interlaboratoires sur les Hydrocarbures Aromatiques Polycycliques (HAP), (French language). LCSQA (convention 04000087): available at http://www.lcsqa.org (anonymous; laboratories numbers available from the authors). 
Table 1

Summary of the different field campaigns: locations, sampling devices used, environmental conditions and number of samples

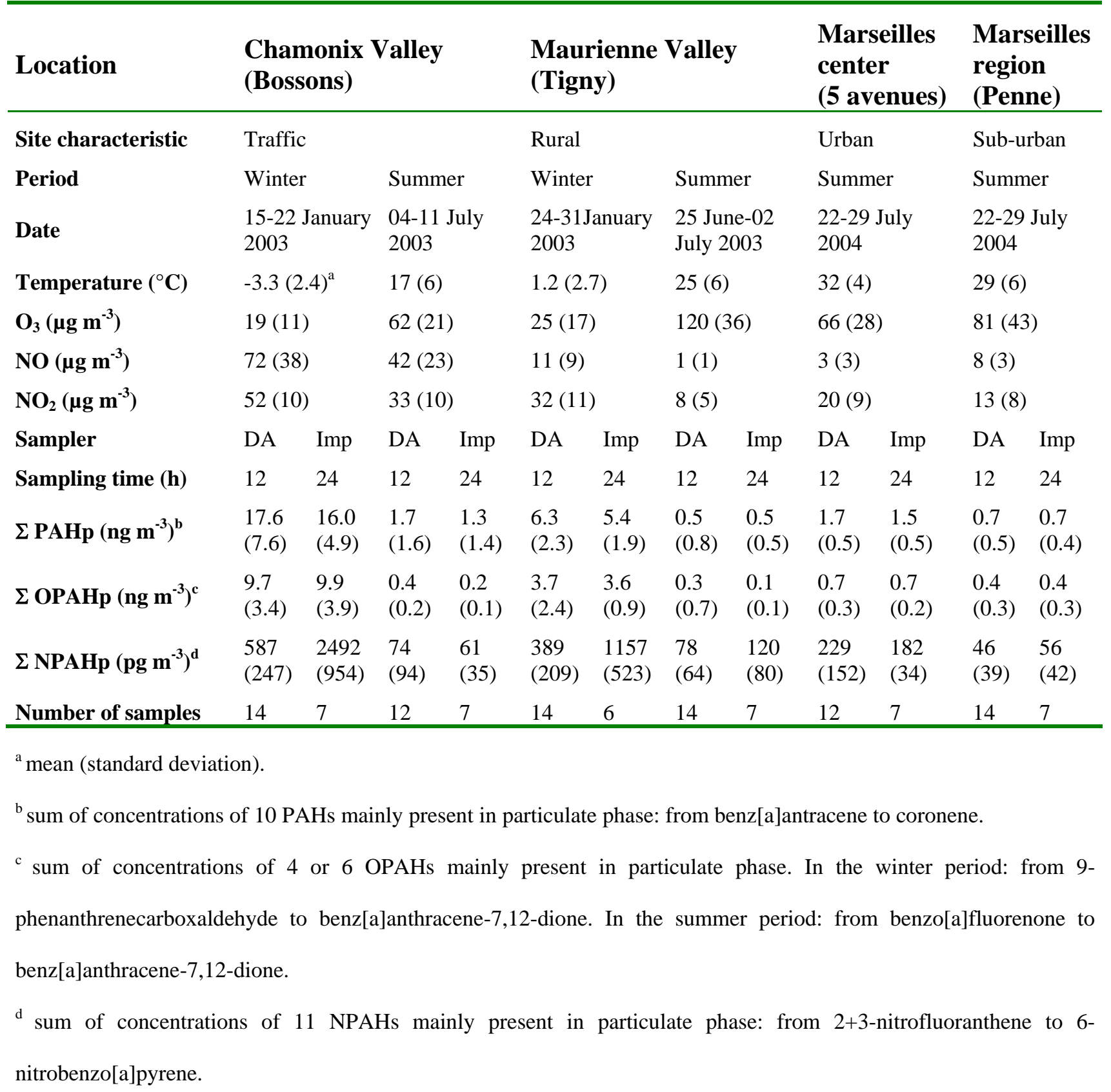




\section{Figure Captions}

Fig. 1. Comparison of particle-bound individual PAH, OPAH and NPAH concentrations measured by $\mathrm{PM}_{10}$ classic filter method (DA-80) and cascade impactor method in the winter and the summer period (sampling sites all mixed).

Fig. 2. Fraction of particle-bound NPAHs collected on the fritted filters and the back up filter according to the sampling campaign.

Fig. 3. PAH, OPAH and NPAH concentration ratios determined by $\mathrm{PM}_{10}$ classic filter method (DA80) and cascade impactor method according to the difference of mean internal temperature of the DA-80 and mean external temperature (all sampling campaigns taking into account). 

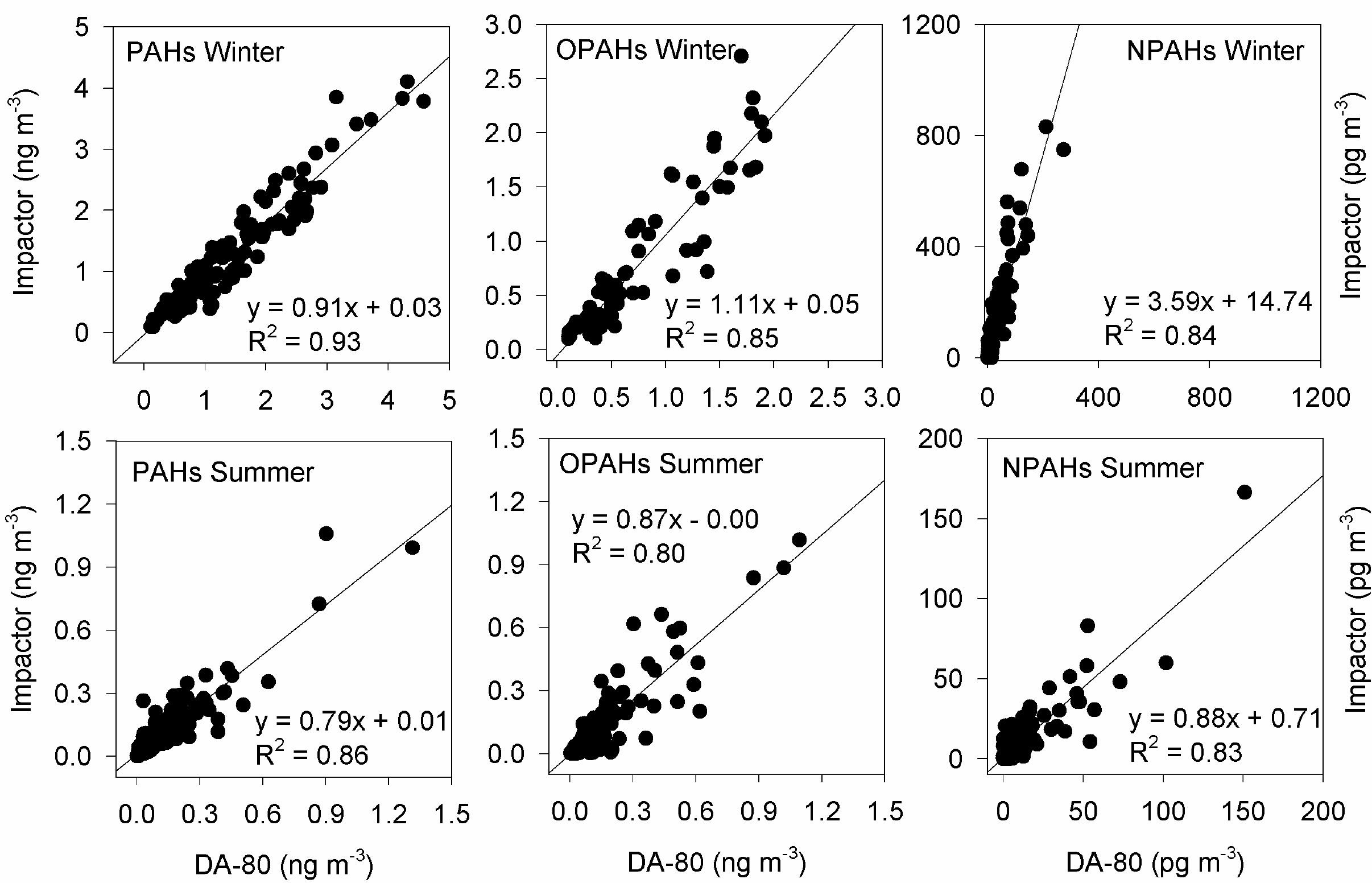


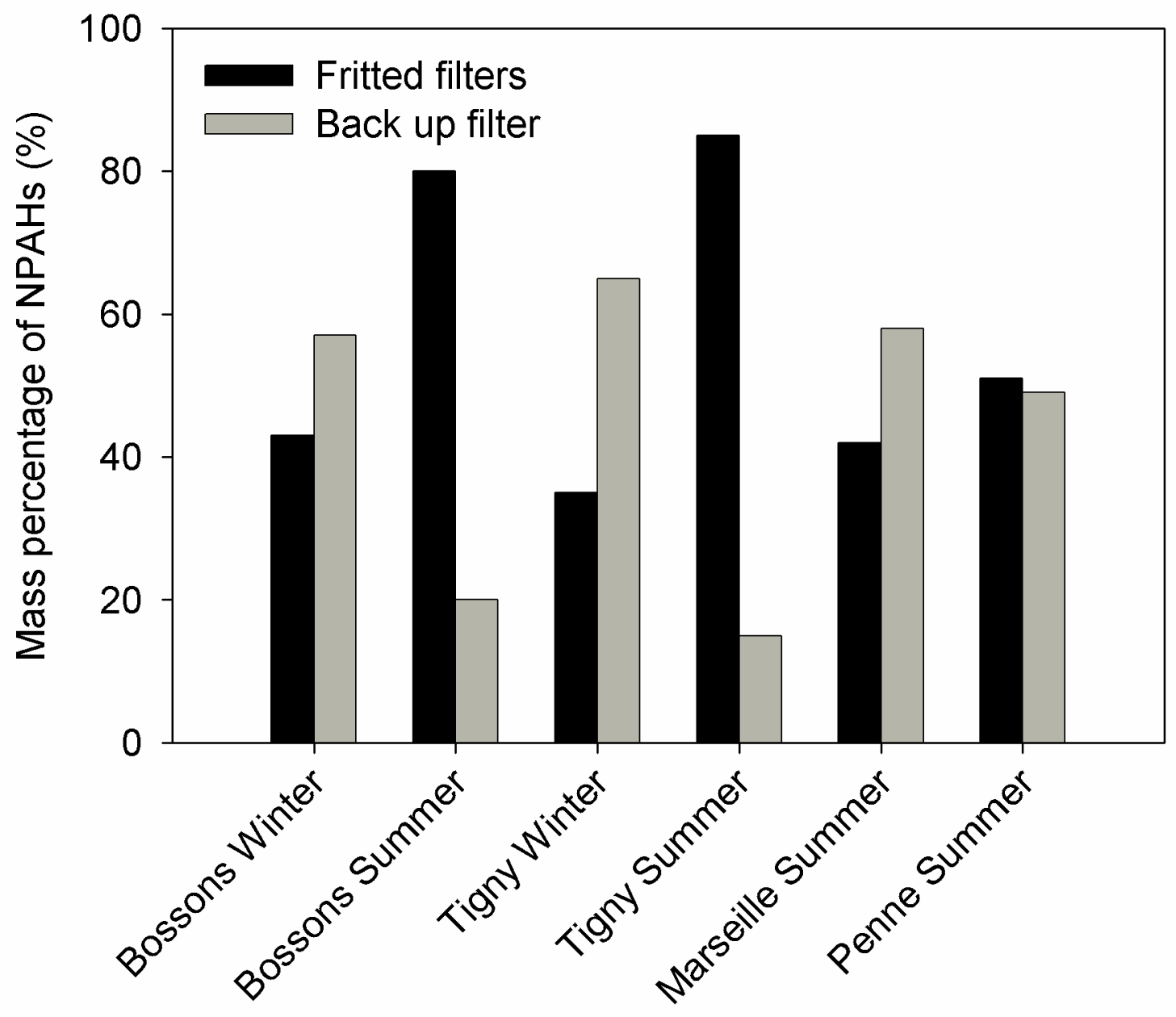


